\title{
Subject index to volume 33
}

Abdomen, acute 704

Absorptiometry, dual energy X-ray 402

Action potentials 346,707

Activities of daily living (ADL), functional levels and 189, 290, 595

Africa 579

Ageing 290, 326, 367, 595

Aneurysm, abdominal aortic 480

Anorectal function 34

Anterior cord syndrome 423

Antibiotics, suppression in neuropathic bladder 156

Anticholinergic agents 274

Aortic occlusion 136

Apnoea, sleep 271

Arachnoiditis ossificans 224

Arm crank 267

Arthritis, rheumatoid 480

Atrophy, cardiac 80

Atropine, intravesical 36

Axon regeneration 381

Baclofen 551, 678

Bedrest, long-term 669

Bladder

biopsy 565

history of catheterisation 429

neurogenic vesical dysfunction 530

recovery following SCI 493

UTI and cystitis in 523

see also Neuropathic bladder

Blood flow 90, 136

Body composition 402

Body fat

distribution of 102

skinfold measurements of 402

Body satisfaction 687

Bone

biochemical markers 669

densitometry 669

injury 573

metabolism 669

mineral 674

scan, for heterotopic ossification 170

Book reviews 57, 176-177, 235-236, 302 , $547,610,741$

Bowel, neuropathic 701

Bowel function after SCI in childhood 701

Brain injury and locked-in syndrome (LIS) 640

Brucellosis 167, 294

Bullet, migrating 541

Calcium crystal deposition in ligamentum flavum 219

Calculi, management by ESWL 132

Cardiovascular disease (CVD), risk factors in SCI 102
Cardiovascular state, and RGO-II orthosis 654

Catheter

history of the 429

indwelling 161

Catheterization

clean intermittent (CIC) 30, 161, 619

in development of management of neuropathic bladder 305

intermittent 69,693

Cauda equina syndrome 219

Cells, arachnoidal 224

Central nervous system (CNS), regeneration in 10

Cerebrospinal fluid (CSF), flow in posttraumatic syringomyelia 203

Cervical spinal cord injury (CSCI)

assessment by MRI 212

clean intermittent catheterisation in 30

from diving 246

minor trauma to neck in patients with OPLL 330

motor paralysis following 506

MRI studies in patients with 573

ossification of posterior longitudinal ligament 18,25

reflex sympathetic dystrophy in 602 upper, neurological deficits 195

vertebromedullary injuries 46

Cervical spine, locked facets in 434

Cervical spondylotic myelopathy (CSM) 354

Chest, pathology and radiographs 121

Children, SCI in 362,701

Cisapride 625

Classification

of pain 555

Zancolli 189

Collagen, injections for intrinsic sphincter deficiency 697

Colon, after SCI 701

Colonization, urethral 69

Community care, in Romania 49

Comparison, of intrathecal baclofen delivery systems 551

Compliance, of clean intermittent catheterization 161

Complications

of clean intermittent catheterization 161

following posterior instrumentation 423

following screw/plate fixation 606

postoperative 538

of SCI in children 362

for SCI patients in a regional population 308

of spinal cord omental transposition 536 and SSCIS level-of-living survey 726 of traumatic SCI, Stockholm Study 585

Computerised tomography (CT), in assessing lung changes 121

Conduction block 354

Constipation

neuropathic 701

and RGO-II orthosis 654

Corticospinal tract, axon degeneration 136

Craniocervical junction 228

CSCI see Cervical spinal cord injury

Cushions, and tissue viability 141

Cyst, bronchogenic 228

Cystometry, following cisapride treatment 625

Cystoplasty, augmentation 560

Decompression

anterior 18

spinal cord 538

Decompression sickness 636

Depression, methodological studies 377

Detrusor, hypercontractility 36

Diapason system 216

Disability, assessment 510, 665

Disc, intervertebral 434

Diving 246

Drugs, intravesical anticholinergic 36

Dysreflexia, autonomic 704

Dystrophy, reflex sympathetic 602

Echocardiography 80

EDRF (endothelium-derived relaxing factor) 450

Ejaculation, seminal somatostatin in 374

Elderly, flowmotion and pressure sore formation in 387

Electrical activity, rectal 346

Electrical stimulation, muscle 437, 514, 707

Electrical stimulation cycle ergometry (ESCE), exercise 80

Electroejaculation 263

Electromyogram, evoked 338

Electromyography, in shoulder dysfunction 148

Electrorectography 346

Embolism, pulmonary 278

Emission, seminal 263

Endothelium-derived relaxing factor (EDRF) 450

Epidemiology of SCI

health related issues 726

in Japan 183

in Novosibirsk, Russia 322

in a regional population 308,726

Stockholm Study 585, 726

in Turkey 469

in USA 62

Epididymitis 619

Erectile dysfunction 77, 628, 731 
Erection, involuntary penile 338

Erosion, vertebral 480

ESCE (electrical stimulation cycle ergometry), exercise 80

Escherichia coli 69

ESWL (extracorporeal shock waves lithotripsy), management of calculi by 132

Evoked potentials

somatosensory 338

spinal cord (SCEPs) 354

Exercise

electrical stimulation cycle ergometry (ESCE) 80

functional electrical stimulation (FES) 90

Exertion, ventilatory thresholds during 419

Extracorporeal shock waves lithotripsy (ESWL), management of calculi by 132

Extremities, upper, peripheral nerve injury to 208

Facets, locked 434

Fatigue, muscle 707

Fertility, influence of bladder management on 263

FES see Functional electrical stimulation

Fever, prolonged, and heterotopic ossification 170

Fixation

plate and screw 606

transpedicular screw $216,350,423$

Flaps, sensory 116

Flexion, response 126

Fluoroscopy, in enteral feeding 153

Fluxmetry, laser Doppler 387

Fracture-dislocation, thoracic, neurological features 734

Fractures

burst 73

spinal 281

thoracolumbar 73

Functional electrical stimulation (FES) 126

exercise 90

restoration of functional gait by 660

RGO-II orthosis and 647

Fusion, posterolateral 216

Gabon, treatment of Pott's paraplegia in 579

Gait

orthosis, reciprocating (RGO) 409, 458 , 647,654

restoration of $126,647,654,660$

Gas tension, transcutaneous 141

Gastrostomy, percutaneous endoscopic (PEG) 153, 416

Goal setting, for spinal lesion levels 189

Graft, spinal cord transplantation 250

Granuloma, solitary eosinophilic 485

Gunshot wounds 54

Hand, functional, limitations 30
Handicap, assessment using Reintegration into Normal Living Index (RNLI) 665

Herniation, lumbar disc 167

Histiocytosis, Langerhans cell 485

Histochemistry, of tibialis anterior muscle 514

History, of bladder catheterization 429

Hormones, antidiuretic 94

Hypotension

acute, associated with terbutaline 300

orthostatic 450

Immobilization, long-term 669

Implants, failures 5

Impotence, management of 77

Incidence of SCI

in Istanbul 469

in Japan 183

in Novosibirsk, Russia 322

in US 62

Incontinence, urinary 530, 560, 697

Index, Wheelchair Users Shoulder Pain (WUSPI) 290, 595

India, SCI centre in 350

Indomethacin, for prolonged fever 170

Injury

brain 640

overuse 296

spinal, from migrating bullet 541

sports 296

thoracolumbar 350

unstable 195

vertebromedullary, prediction of outcome of 46

Instrumentation, spinal 281, 423

Ipratropium, bronchodilatory effects of 274

Ischaemia, spinal cord 136

Istanbul, SCI in 469

Japan, epidemiological survey of SCI 183

Kyphosis 281

Labelling, MIB- 1 and PCNA 523

Laminoplasty, for myeloradiculopathy 25

Length of stay (LOS), of ASCI patients 254

Lesions, spinal 294

Life quality 555

Life satisfaction 334

Ligamentum flavum, calcium deposition in 219

Lipoproteins 102

Lithiasis, urinary 132

Lithotripsy, extracorporeal shock waves (ESWL), management of calculi by 132

Locked-in syndrome (LIS), motor control in 640

Locomotion

efficiency of reciprocating gait orthosis (RGO) 409

paraplegic 660

Lumbar spine 216, 485
Lumbosacral spine

spontaneous migration of bullet to 541

transpedicular screw fixation 216

Lungs, effects of ipratropium bromide on 274

Lymph vessels 472

Magnetic resonance imaging (MRI) assessment of CSCI 212

in ossification of posterior longitudinal ligament 18

in post-traumatic syringomyelia 203

studies in patients with CSCI 573

study of brucellar spondylitis 167

Manometry 40, 346

Metabolism

bone 669

calcium-phosphorus 164

Metaplasia

mesenchymal 224

osseous 543

Methylprednisolone, neurological outcomes 316

MIB-1 labelling 523

Micturition, automatic 493

Morphology

of lymphatic microcirculation 472

of tibialis anterior muscle 514

Mortality rate for ASCI patients 254

Motility, rectal 346

Motor control, in locked-in syndrome (LIS) 640

Motor level (ML) 510

Motor recovery, following SCI from stab wounds 98

Mouse, CEA-transgenic 381

MRI see Magnetic resonance imaging

Muscle

quadriceps 707

scapular retractor 148

skeletal 437,514

smooth 346

torque output 707

trauma and heterotopic ossification 464

Myelopathy

cervical spondylotic (CSM) 354

cystic, of thoracic spinal cord 538

Myeloradiculopathy 18, 25

Myoclonus, propriospinal 678

Neck, minor trauma to 330

Nerve, regeneration 359

Nerve roots

spinal, repair 359

ventral, implantation 359

Nerves, sacral, dysfunction in lumbar spinal stenosis 338

Neurological deficit 73

following upper cervical spine injury 195

from migrating bullet 541

Neurological deterioration

in SCI patients in a regional population 308

in traumatic SCI 585

Neurological impairment, resulting from 
rhabdomyolysis and heterotopic ossification 164

Neurological level (NL) 510

Neurology, lower urinary tract function 493

Neuropathic bladder

augmentation cystoplasty for 560

clean intermittent catheterization in 161

cutaneous ileocystostomy for vesical dysfunction 530

detrusor hypercontractility in 36

development of management of 305

effect of cisapride treatment on 625

intrinsic sphincter deficiency in 697

management, influence on fertility 263

risk of vesical neoplasia in 565

spinal reflex bladder 40

suppressive antibiotic therapy in 156 see also Bladder

Neuroplasticity, lower urinary tract 493

Neurotrophic factors 359

Novosibirsk, epidemiology of SCI in 322

Nutrition, enteral 153

Obesity, in men with SCI 102

Obstruction, bronchial 274

Oliguria, after SCI 721

OPLL see Ossification

Orthoses

halo cervical 110

reciprocating gait (RGO) 409, 458, 647, 654

Osmolality, serum and urine 94

Ossification

heterotopic

biochemical analysis 398

early diagnosis of 464

of lumbosacral dura and arachnoid

$$
543
$$

and muscle trauma 464

and prolonged fever 170

rhabdomyolysis and 164

intraspinal 543

of posterior longitudinal ligament

(OPLL) 18, 25, 330

Osteophyte, formation after decompression 538

Osteoporosis 281

disuse 669,674

and RGO-II orthosis 654

Osteosynthesis, anterior cervical 606

Outcomes

long-term 367,665

neurological 316,330

for SCI patients in a regional population 308, 726

for traumatic SCI, Stockholm Study 585

Oxygen

consumption 267

saturation 271

Pain

psychological distress 555

shoulder 290, 595

Papaverine, in management of impotence
77

Paralysis

lower limb 90

motor, neurological recovery 506

spinal 551

Paraparesis 224, 281, 480

Paraplegia

brucellosis in 294

cystic myelopathy after decompressive surgery 538

and decompression sickness 636

electrical stimulation of tibialis anterior muscle in 514

exercise responses of men with 267

following aortic occlusion 136

motor recovery in 98

muscle fatigue in 707

oliguria in 721

peripheral nerve injury in 208

Pott's 579

pulmonary embolism in 278

restoration of functional gait by FES 660

restoration of muscle contractile properties 437

restoration of sensation in 116

risk of vesical neoplasia in 565

sexual dysfunctions in men with 628

and shoulder pain 290,595

tarsal tunnel syndrome in 482

thromboembolic disease in 472

tissue viability in 141

use of reciprocating gait orthosis (RGO) in 409,458

ventilatory thresholds during wheelchair exercise 419

Paresis, following upper cervical spine injury 195

PCNA labelling 523

Perforation

in acute abdomen 704

oesophageal 606

Peripheral nervous system (PNS), injury to 208

Polymerase chain reaction (PCR), investigation of transplanted peripheral nerves 381

Polyuria, and ADH levels 94

Posterior longitudinal ligament, ossification of (OPLL) 18, 25, 330

Potassium, total body 402

Pott's paraplegia/tetraplegia 579

Pressure sores 141, 387

Prevalence of SCI

in US 62

from diving 246

in Japan 183

Priapism, in lumbar spinal stenosis 338

Procreation, effect of epididymitis on 619

Prognosis

of CSCI and MRI 212

neurological, following upper cervical spine injury 195

thoracic fracture-dislocation 734

Prony spectral line estimation (PSLE) 387

Prostaglandin, E1 intracavernous injection
731

Pulmonary function testing 274,454

Pumps 551

Quality of life, effects of ageing and durability of disability on 367

Radicals, superoxide 450

Radiculopathy, lumbar 219

Radiographs, chest 121

Recovery, neurological, of ASCI patients 254

Rectum, electrorectogram study of neuropathic 346

Reflex, flexor-withdrawal 482

Reflex sympathetic dystrophy (RSD) 602, 715

Reflux, vesicoureteral 560

Regeneration

axonal 381

in central nervous system 10

Rehabilitation 189,458

Reintegration, social 49

Research, spinal cord injury (SCI) 10

Reservoir, urinary 560

Respiratory distress 195

Reviews, experimental spinal cord transplantation 250

Rhabdomyolysis, and heterotopic ossification 164

Risk factors, heterotopic ossification 464

Romania, survey of SCI in 49

Russia, epidemiology of SCI in 322

Sacral anterior root stimulator 5

Screw 606

transpedicular 216, 350, 423

Semen, somatostatin concentration 374

Sexuality

affective state 687

body satisfaction 687

dysfunctions in SCI men 628

female 687

management of impotence 77

in people with SCI 334

Shock, spinal 300

Shoulder dysfunction 148

Shoulder pain measurement 290, 595

Shoulder-hand syndrome 602

Shunt, syringo-peritoneal 241

Skin response, sympathetic (SSR) 602

Sleep

apnoea 271

and locked-in syndrome (LIS) 640

registration of nocturnal 271

Slovenia, prevention of SCI from diving in 246

Somatostatin, concentration in semen 374

Sounds 429

Spasm, relief by intrathecal baclofen 551

Spasticity

and myoclonus 678

relief of, by intrathecal baclofen 551

Sphincter

artificial urinary 560

intrinsic deficiency 697 
Spinal canal, spontaneous migration of bullet to 541

Spinal cord

cavitation 538

and decompression sickness 636

evoked potentials (SCEPs) 354

experimental transplantation of 250

omental transposition 536

regeneration 250

Spinal cord injury (SCI)

acute abdomen in 704

acute hypotension associated with terbutaline in 300

$\mathrm{ADH}$ levels and polyuria in 94

adiposity in men with 102

aetiology 183

biochemical analysis of heterotopic ossification 398

body composition in individuals with 402

bone matabolism in 669

bone mineral measurement in 674

calculi management by ESWL in 132

in children 362,701

depression in 377

development of management of neuropathic bladder 305

early surgery for thoracolumbar 350

effects of ipratropium bromide in 274

effects of methylprednisolone in 316

erectile dysfunction in $77,628,731$

experimental 450

from stab wounds 98

implant failures 5

intrathecal baclofen delivery systems 551

level-of-living survey 726

long-term outcomes of 367,665

medical problems in a regional population 308

medico-social survey of Romanians with 49

muscle trauma and heterotopic ossification in 464

neurogenic vesical dysfunction 530

neurological level 263, 510

neurological recovery 254

oliguria after 721

ossification of lumbosacral dura and arachnoid following 543

pain and life quality within 2 years of 555

peripheral nerve injury in 208

post-traumatic syringomyelia in 203, 241

pressure sore formation in 387

prevalence in US 62

psychological problems in 377

pulmonary embolism in 278

pulmonary function testing in 454

recovery of lower urinary tract function 493

reflex sympathetic dystrophy (RSD) in 715

researches 10

restoration of functional gait in 647 ,
654,660

restoration of gait by FES 126

risk of vesical neoplasia in 565

seminal somatostatin in men with 374

sexual dysfunctions in men with 628

sexual function in women with 687

sexuality in 334

spinal reflex bladder in 40

Stockholm Stuciy 585, 726

superoxide radicals in 450

suppressive antibiotic therapy in 156

treatment of sleep apnoea in 271

tympanic temperature measurement in 476

urinary tract changes with ageing 326

urinary tract infection (UTI) in 523

urological management of 619

vertebromedullary 46

vesicourethral and anorectal function in 34

Wheelchair User's Shoulder Pain Index (WUSPI) 290, 595

see also Cervical spinal cord injury;

Spine Epidemiology of SCl

acute injury 121

fracture 281

stabilization 110

Spondylitis, brucellar 167

Spondylosis, cervical 354

Sports, ventilatory thresholds during 419

Stab wounds, motor recovery following 98

Standing, restoration of, by FES 126

Stenosis, lumbar spinal 219, 338

Stockholm Spinal Cord Injury Study (SSCIS) 585, 726

Stomach, volvulus 536

Strength activities, remedial 148

Stricture, urethral 619

Superoxide radicals 450

Surgery, early, for thoracolumbar SCI 350

Surveys

level-of-living 726

medico-social 49

see also Epidemiology of SCI

Syringomyelia 482

post-traumatic 203, 241

Tachycardia, supraventricular 278

Tarsal tunnel syndrome 482

Temperature

oral 476

rectal 476

tympanic 476

Terbutaline, acute hypotension associated with 300

Tetraparesis 354

Tetraplegia

acute hypotension associated with terbutaline in 300

brucellosis in 294

and decompression sickness 636

effects of ipratropium bromide in 274

electrical stimulation cycle ergometry
(ESCE) exercise 80

from diving injury 246

functional levels and movement in 189

heterotopic ossification in 170

and locked-in syndrome (LIS) 640

motor and neurological levels in 510

motor recovery in 98

myoclonus in 678

oliguria in 721

Pott's 579

reflex sympathetic dystrophy (RSD) in 602

risk of vesical neoplasia in 565

sleep apnoea in 271

tympanic temperature measurement in 476

ventilatory thresholds during wheelchair exercise 419

Thoracic spinal cord, cystic myelopathy of 538

Thoracic spinal cord injury, exercise responses of men with 267

Thromboembolic disease 472

Tissue

composition 674

viability in SCI 141

Torque, output of muscle 707

Transplantation, experimental, of spinal cord 250

Transposition, omental 536

Trauma

conservative treatment of 73

muscle 464

penetrating 98

spinal, locked facets 434

to neck 330

Treatment

of brucellosis 294

conservative, of thoracolumbar fractures 73

medical-surgical, of Pott's paraplegia 579

methylprednisolone in SCI 316

non-operative, for thoracic fracturedislocation 734

Trimethoprim-sulphamethoxazole prophylaxis against UTI 156

Tuberculosis, spinal 579

Tumour

extramedullary 228

spinal 485

Turkey, SCI in 469

Ulcer, decubitus, formation 387

Ulceration, sacral pressure 116

Ultrasonography 464, 693

Urethra

neuropathic 697

urinary leakage from 530

Urinary tract, changes with ageing 326

Urinary tract infection (UTI) $69,156,523$

Urine, residual 693

Urodynamics 40,530

Urology, development of management of neuropathic bladder 305 
Vasomotion, and pressure sore formation 387

Ventilation, thresholds during wheelchair exercise 419

Vertebrae, cervical 506

Vesicourethral function 34

Vibroejaculation 263

Vital capacity 454

Volvulus in acute abdomen 704 stomach 536

Weightlessness 669

Wheelchair

exercise 419

marathon 208

racing 296

users 148 propulsion 267

Shoulder Pain Index (WUSPI) 290, 595

Working ability 458

Wounds

gunshot 541

stab 98

Zancolli classification 189 